\title{
Characterization and Authentication of Probiotic Preparations
}

\author{
Vijay Kothari ${ }^{\mathrm{a}^{*}}$, Anselm de souza ${ }^{\mathrm{b}}$, Dilip Mehta ${ }^{\mathrm{b}}$

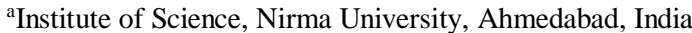 \\ ${ }^{\text {b}}$ Viridis Biopharma Pvt. Ltd., Mumbai, India \\ *vijay.kothari@nirmauni.ac.in; vijay23112004@yahoo.co.in
}

Probiotics are the living microorganisms, administered for providing beneficial health effects to the consumer. Numerous reports describing the important role of indigenous microbiota in maintaining human health are being added to the already existing body of scientific literature. Human microbiota is being shown to have significant correlation with conditions like obesity, diabetes, neurological disorders, and even mood and behaviour. Microbial inhabitants of humans, their ecology, and their role in human health and disease has already become a hot research topic. The communication between human brain and gut biota through the gut-brain axis is being shown to be of central importance in overall maintenance of human health and immunity [Mayor et al., 2015], and also deciding susceptibility of the human system to various disease conditions, which were previously thought to be purely of physiological, neurological or metabolic origin with no visible microbial connection. In this background, manipulation of the indigenous microbiota through various strategies such as use of prebiotics and probiotics is likely to gain more and more prominence from a nutraceutical and therapeutic point of view.

A good number of probiotic formulations have been introduced in market, and many more are likely to be introduced in near future. These commercial probiotic formulations contain one or more of microbial strain(s), and some of them even claim particular health benefit(s). An aware consumer and concerned regulatory authorities can have many questions regarding such commercial probiotic preparations in market, such as:

- Whether the product contains that many number of bacteria as written on the product label

- Whether the product contains the same species and/or strain of bacteria, as described on its pack

- Does the number of bacteria indicated on the product label remain same since production, through storage, to consumption

- Can the bacteria multiply inside the product container during storage, and thus increase their own number? (as many of the probiotic products of dairy origin, they add probiotic bacteria in a nutrient-rich matrix, viz. sweetened milk, ice-cream, etc.)

- Whether the product really provides the type of health benefits as claimed by the producer

- How much quantity of the probiotic product should be consumed at what frequency to receive the claimed health benefit

- Have these probiotic products been assayed by any independent lab for its bacterial content and claimed benefits?

- Can the product contain any microbes not listed on the label by the manufacturer?

To address all such consumer concerns, any commercial probiotic preparation should be subjected to appropriate characterization on the following parameters, each of which is discussed individually in the text below:

A. Genus, species, and strain identity of the process organism

B. Microbial load (per $\mathrm{mL}$ or $\mathrm{g}$ ) of the product at the time of manufacturing, and at the end of shelf-life

C. Biological effect 


\section{A. Genus, species, and strain identity of the process organism}

All probiotic products must contain above information on their label, which needs to be verified in their in-house lab as well as by independent labs. Ensuring accurate and precise labelling assumes even more importance in light of the fact that in past, description written on the product label regarding species identity have been shown not to match with that found by independent investigations. Inaccurate labelling of probiotic species is not uncommon with commercial products [Yeung et al., 2002]. Such inaccuracies can be of two types. First, in which the product actually contains a species different than what is labelled on it. Second, in which the product contains additional (contaminating) species not intended/ claimed by the manufacturer to be part of that particular product. If these unintended strains belong to some pathogenic species, then it becomes a serious issue. 14 out of 29 commercial strains were found by Yeung et al (2002) to carry a species identity different than that mentioned on the product. Hamilton-Miller et al. (1996) reported presence of unlabeled Enterococcus populations in commercial probiotic products. It should be noted that Enterococci may possess hemolytic ability, as well as genes for antibiotic-resistance. Any probiotic product containing such potentially infectious species can not qualify as an ideal product.

Taxonomic investigation on any probiotic product are of utmost importance, considering the fact that probiotic traits are not just species-specific, but they in fact are strain-specific [Weiss et al., 2005]. Different strains belonging to the same genus and species can not provide the same benefit to the consumer. A given health benefit can be linked only to a particular strain, and not all strains of a given species. For example, Lactobacillus rhamnosus GG is considered as a probiotic strain suitable for gastrointestinal health, whereas L. rhamnosus GR-1 is considered suitable for use as probiotic for the prevention of urogenital tract infections [Wilson, 2005]. This strain-specificity makes establishing an unequivocal taxonomic identity of any probiotic organism, very much necessary [Donelli et al., 2013].

There are many methods available for identification of a microbial species, all of them not being equally suitable for different group of microorganisms. Below, a short overview of the methods, particularly suitable for bacterial identification is presented, as most probiotic products contain bacterial species, and among them majority contain strains belonging to the genera Bifidobacterium and Lactobacillus. Though genotyping methods in general seem to offer high degree of specificity, they alone can not be adopted at the cost of conventional or modern phenotyping methods. A polyphasic taxonomic approach is required to obtain most accurate results [Donelli et al., 2013].

- Conventional methods:

Bacteriophage typing and Serotyping are the traditional methods of strain typing, still of use, but largely for pathogenic organisms. Another conventional approach is to rely on the carbohydrate fermentation profile for bacterial identification. However, classical methods of bacterial identification based on the phenotypic characteristics, like the API 50CHL test, may not be fully satisfactory. Boyd et al. (2005) demonstrated that the API 50CHL test could correctly identify only 4 isolates out of a total of 90 isolates from the female genital tracts.

Though not scoring very high on specificity, basic traits such as gram-staining, colony morphology, growth pattern in liquid media, morphology under microscope, routine biochemical tests, etc. should also be given due importance while characterizing probiotic strains. 
- Molecular/ Genetic methods:

Among a variety of available molecular methods, Pulsed Field Gel Electrophoresis (PFGE) has been considered to be a gold standard for bacterial strain typing, and is claimed to be useful for typing a broader array of bacterial species [Tenover et al., 1995]. In addition to PFGE, methods like FISH (Fluorescence In Situ Hybridization) and RAPD (Random Amplification of Polymorphic DNA) have also been applied for differentiation of Lactobacillus species. All such methods were developed for general taxonomical purposes, however their standardization for the probiotic strains is needed to make them most appropriate for strain identification of Lactobacillus and Bifidobacterium [Gosiewski et al., 2012]. MLST (Multilocus Sequence Typing) is another method of relevance. While practicing methods like RAPD, selection of the most discriminative primer needs to be done to get the most accurate strain differentiation. Methods like RAPD and PFGE does not seem to be widely practiced for the probiotic bacteria. Though cheaper and easier, the RAPD method was reported by Gosiewski et al. (2012) to be inferior to PFGE, with respect to repeatability. RAPD offered a lower degree of differentiation among the strains of Lactobacillus fermentum and L. gasseri than the PFGE method. They reported RAPD and MLST to be less efficient in differentiating particularly the strains of L. plantarum of human origin. Weiss et al. (2005) reported RAPDPCR (randomly amplified polymorphic DNA-polymerase chain reaction) not to be a robust method for $L$. reuteri strain differentiation. Despite its complexity and costs, PFGE method was shown by them to be robust and most suitable for the differentiation and identification of $L$. reuteri strains.

16S RNA sequencing by far is currently the most widely used method for bacterial identification. However, it should be kept in mind that this method is based on sequencing of a very very small part of the whole bacterial genome, and hence may not always be capable of differentiating at the strain level. This and any other methods relying on universal primers (and not species/ strain specific primers) will always score less than cent percent when applied to strain differentiation. Designing strain-specific primers for all major probiotic strains will definitely improve the situation notably.

Many of the molecular methods for bacterial strain typing rely on the restriction patterns. Here, selection of the most appropriate restriction endonuclease becomes of utmost importance. For example, SmaI restriction enzyme has been indicated to be suitable for genotyping of L. gasseri isolates [Kawase et al. 2011]. High degree of homogeneity among some of the probiotic bacteria like L. plantarum makes their strain-typing even more challenging. Within the Lactobacillus plantarum species, one may have to deal with a rare microbiological phenomenon concerning the formation of the genetically stable clones, as described by Markiewicz et al. (2010). Their genotyping may require use of alternative methods like whole genome sequencing, or MLVA (Multiple Locus Variable number tandem repeat Analysis).

Modifications of existing methods for bacterial strain-typing keep appearing. For example, Yasuda and Shiaris (2004) reported a method for differentiation of bacterial strains by thermal gradient gel electrophoresis, putting into use the non-GC-Clamped PCR primers for the 16S-23S rDNA intergenic spacer region. Few of the existing classical or molecular methods being used in general for bacteriotyping are reported not to be that useful for probiotic bacteria. For instance, carbohydrate fermentation analysis was not found to be sensitive enough for identification of species within the L. acidophilus group. Fatty acid methyl ester (FAME) 
analysis has also been shown to be inaccurate and not that reliable for the identification of lactobacilli [Donelli et al., 2013].

In addition to various genotyping and phenotyping methods described above spectral fingerprinting may also emerge in near future as a reliable novel method for bacterial strain typing. Spectral fingerprinting may be developed as a cultivation-free method. For example, Raman spectroscopy is currently being mentioned to possess all of the features needed to characterize and identify bacteria [Lorenz et al., 2017]. It is a noninvasive technique, which may well complement established microbiological and molecular methods. Spectral fingerprinting can yield useful information from even single bacteria. Further improvements can be achieved by combining Raman spectroscopy with stable isotope probing to investigate microbial interactions. With appropriate statistical analysis applied, Raman spectroscopy is expected to allow identification of bacteria at different taxonomic levels.

Almost all bacterial identification schemes compare the genetic, morphological, biochemical, and physiological profile of the test strain against those of the bacteria already listed in some international database. This simply means that accuracy and reliability of identification and differentiation of microbial species directly depends on accuracy and coverage of the widely used databases of genomic, transcriptomic, or metabolomic data. Continuous updation and refinement of all such major international databases, particularly with respect to major probiotic strains, is necessary.

\section{B. Microbial load of the product at the time of manufacturing, and at the end of shelf-life}

Strength of any probiotic product is directly dependent on the number of microorganisms present per unit volume or weight of the product. Many of the probiotic products do mention the minimum number of spores or CFU present in individual pack of the product. However, this number may change over the course of transport and storage of the product, wherein a suitable temp always may not be maintained that strictly. Since these products contain live organisms, or the spores capable of germination, these cells or spores may get inactivated owing to a variety of factors. Most of these commercial products mix probiotic cells with some matrix material of edible nature, and thus it is not out of place to ask whether the cells can replicate inside the product container using the edible matrix as a source of nutrition. If this happens, then it will have a direct bearing on the recommended dose-size of the probiotic product. It is not impossible for the microbial cell/ spore count at the time of manufacturing to vary from that at the end of shelf-life.

In the above context applying the most robust enumeration techniques becomes important for accurately quantifying the microbial load of a given probiotic formulation. Among the major methods available for bacterial enumeration are:

- Plate count method

- Microscopic count

- Electronic particle counter

- Flow cytometry

Plate count method is the most widely used method for determining the Colony Forming Units $(\mathrm{CFU})$ in a given sample. Though quite useful, this method has few inherent limitations. Results of the plate count method vary widely depending on composition of the medium used for cultivation, incubation conditions, duration of incubation, etc. Results of this method often do not match with 
those of microscopic count, the latter being on higher side. This is famously referred to as great plate count anomaly. Electronic particle counters though do not suffer from the manual errors (as possible with plate count), they can not distinguish dead cells from the live cells. Inability of distinguishing live cells from dead cells is also there with the microscopic counts. Methods relying on conversion of substances like MTT (a tetrazolim salt) by living bacteria from their oxidized to reduced form are also relevant, as intensity of the colour change is linked with the level of metabolic activity of the bacterial culture being investigated [Zhang and Liu, 2002]. Standard curves correlating OD at an appropriate wavelength with bacterial load, specifically constructed for probiotic species can also be used.

Quantification of bacterial viability can also be achieved by flow cytometry. Though it yields rapid results, and different stains are available to establish viability, staining protocols are not that consistent. A general optimization approach is lacking for microbiological applications. Discrepancy does exist between protocols with respect to cell-permeant nucleic acid and functional stains. As different dyes may behave differently within a particular combination of medium matrix, microorganism, and instrument, protocols need to be optimized to obtain reproducible results [Buysschaert et al., 2016]. A flow cytometry method to assess the functionality of different populations in fermentation starters and probiotic products has been reported by Bunthof and Abee (2002). Davis (2014) has reviewed various culture-dependent and alternative techniques for quantification of viable bacteria, particularly in context of probiotic strains.

It should be noted that it is possible for the live probiotic bacteria to exist in a viable but nonculturable (VNC) state. Cell culture techniques can only estimate numbers of replicating strains but not those lying in VNC state. This situation demands for an operating definition of live probiotic bacteria. Culture-independent techniques should be exploited along with traditional culturedependent techniques for more accurate enumeration of viable probiotic strains. Investigation of same probiotic product subjected to different methods of enumeration will yield varying results, and thus some logical consensus needs to be built regarding the most appropriate methods of enumeration of probiotic preparations. Methods capable of focusing on living cells seem to be most relevant here, as probiotic strains are ideally expected to reach live and intact in the human consumer's gut.

\section{Biological effect}

Utility of any probiotic preparation can be considered high, only if it can be demonstrated to exert desired biological effect in the human host. Both in vitro and in vivo (animal trials as well as human trials) assays should be used to demonstrate the biological efficacy of a probiotic strain. The possible beneficial effects expected from different probiotic strains may include (Wilson, 2005):

- Preventing colonization of human surfaces by exogenous pathogens

- Displacement of a pathogen already established on a particular body site

- Providing for metabolic activities not present in the host

- Immune system stimulation

A particular probiotic strain obviously can not be expected or tested for all such beneficial traits. Tests to be performed for the given probiotic strain will be defined by its intended use, i.e. whether it is to be used for gastrointestinal (GI) tract, vaginal tract, or oral cavity. For example, properties for which probiotic strains claimed to be capable of preventing urogenital tract infections are to be tested, may include: 
- Ability to colonise the vaginal tract after being administered orally

- Tolerance to acid and bile

- Lack of pathogenicity and carcinogenicity

- Production of substances exerting antagonistic effect on exogenous microbes

- Ability to prevent adhesion of exogenous microbes to epithelial cells

- Ability to adhere themselves to the epithelial cells

- Genotypic and phenotypic profile matching to a high degree with the natural isolates from urogenital tract of healthy females

Similarly, the probiotic strains claimed to be beneficial for GI tract may be assayed for some of the following properties:

- Ability to decrease the course of antibiotic-induced diarrhoea

- Ability to decrease duration of diarrhoea in Clostridium difficile-associaed colitis

- Ability to protect against traveller's diarrhoea, or rotavirus-induced diarrhoea

- Ability to confer protection against asthma on infants, when administered to mothers during and post-pregnancy

- Ability to reduce colonization of stomach by Helicobacter pylori

- Ability to reduce relapses in patients with Crohn's disease (relevant for organisms like Saccharomyces boulardii)

- Ability to reduce symptoms of irritable bowel disease

- Ability to increase the production of anti-inflammatory cytokines, and/or to decrease the production of pro-inflammatory cytokines

For testing the in vivo biological efficacy of probiotic strains, besides using cell lines, and conventional animal models, simpler life forms like Caenorhabditis elegans, Drosophila melanogaster, zebrafish, etc. can also be employed. Though these simpler models can not replace the animal models like mice, they can certainly help in reducing the sacrifice of more complex experimental animals. Use of simpler model organisms like $C$. elegans can help in screening out the less promising candidates at earlier stages. C. elegans can be infected successfully by many human pathogenic bacteria including Pseudomonas aeruginosa, Staphylococcus aureus, etc. [Sorathia and Rajadhyakha, 2016]. Ability of probiotic strains to confer protection against such pathogenic strains can be verified in this simple nematode worm. Use of such simpler animal model hosts is likely to raise no major ethical concerns.

\section{Final Comments}

With the phenomenon of gut dysbiosis getting more and more attention from medical professionals and scientists, the probiotic products are very much likely to be prescribed more and more. Increasing number of cesarean births and lesser preference for breast feeding among modern mothers are factors making a large number of newborns devoid of adequate natural flora, who are amenable for manipulation of their flora through the probiotic route. With such an optimistic scenario for the probiotic industry, a strong need for regulation of the probiotic products is being felt. Whatever few guidelines [Pineiro and Stanton, 2007; Ganguly et al., 2011] from governmental or non-governmental sources on probiotic products are available, they all emphasize on thorough characterization and proper labelling of the probiotic products. However, no standardized protocols acceptable at international level have been made available for this purpose. At present there is no consensus on which are the best 
methods for strain typing of the probiotic microorganisms, and for accurate quantification of viability and functionality of the probiotic strains. Current literature indicates PFGE to be the most suitable method for strain-typing of probiotic bacteria like Lactobacillus, whereas carbohydrate fermentation profile and FAME have been indicated not to be that useful and reliable. A polyphasic taxonomic approach is recommended by almost all experts for more reliable strain-typing, rather than relying solely on phenotyping or genotyping. Newer methods like spectral fingerprinting are likely to make the microbiologist better equipped for probiotic strain differentiation. Same can be said regarding flow cytometry for enumeration purpose. Among all different parameters such as strain authentication, safety assessment, microbial load quantification, efficacy claims, etc., accurate strain differentiation of the probiotic strains seem to be the most challenging to be performed with high accuracy. Future developments for better evaluation and characterization of probiotic products will certainly help the probiotic industry to present their products to the consumers in a more reliable and confident fashion.

\section{References}

Agnes Weiss, Hans Peter Lettner, Walter Kramer, Helmut Karl Mayer, Wolfgang Kneifel (2005). Identification of Probiotic Lactobacillus reuteri Strains, Food Technol. Biotechnol., 43 (3) 295-300.

Boyd MA, Antonio MA, Hillier SL (2005) Comparison of API $50 \mathrm{CH}$ strips to whole-chromosomal DNA probes for identification of Lactobacillus species. J Clin Microbiol 43:5309-5311.

Bunthof, C. J., \& Abee, T. (2002). Development of a Flow Cytometric Method To Analyze Subpopulations of Bacteria in Probiotic Products and Dairy Starters. Applied and Environmental Microbiology, 68(6), 2934-2942. http://doi.org/10.1128/AEM.68.6.2934-2942.2002.

Buysschaert, B., Byloos, B., Leys, N. et al. (2016). Appl Microbiol Biotechnol,100: 9037. doi:10.1007/s00253-016-7837-5.

Davis, C. (2014). Enumeration of probiotic strains: review of culture-dependent and alternative techniques to quantify viable bacteria. Journal of Microbiological Methods, 103, 9-17.

Fred Tenover, Robert Arbeit, Richard Goering, Patricia Mickelsen, Barbara Murray, David Persing, Bala Swaminathan (1995). Interpreting Chromosomal DNA Restriction Patterns Produced by PulsedField Gel Electrophoresis: Criteria for Bacterial Strain Typing. Journal of Clinical Microbiology, 22332239.

Ganguly, N. K., Bhattacharya, S. K., Sesikeran, B., Nair, G. B., Ramakrishna, B. S., Sachdev, H. P. S., ... \& Katoch, V. M. (2011). ICMR-DBT guidelines for evaluation of probiotics in food. Indian Journal of Medical Research, 134(1), 22.

Gianfranco Donelli, Claudia Vuotto, Paola Mastromarino (2013). Phenotyping and genotyping are both essential to identify and classify a probiotic microorganism. Microbial Ecology in Health \& Disease, 24: 20105 - http://dx.doi.org/10.3402/mehd.v24i0.20105.

Hamilton-Miller, JMTS Shah, CT Smith (1996). "Probiotic" remedies are not what they seem. Br. Med. J., 312:55-56.

Kawase M, He F, Kubota A, Miyazawa K, Yoda K, Hiramatsu M (2011). Strain-specific detection by pulsed-field gel electrophoresis of Lactobacillus gasseri TMC0356 in human feces after oral administration of these organisms. Microbiol Immunol, 55:589-594. 
Lorenz, B., Wichmann, C., Stöckel, S., Rösch, P., \& Popp, J. (2017). Cultivation-Free Raman Spectroscopic Investigations of Bacteria. Trends in Microbiology.

Markiewicz LH, Biedrzycka E, Wasilewska E, Bielecka M (2010) Rapid molecular identification and characteristics of Lactobacillus strains. Folia Microbiol, 55:481-488.

Mayer, E. A., Tillisch, K., \& Gupta, A. (2015). Gut/brain axis and the microbiota. The Journal of clinical investigation, 125(3), 926-938.

Pineiro, M., \& Stanton, C. (2007). Probiotic bacteria: legislative framework-requirements to evidence basis. The Journal of nutrition, 137(3), 850S-853S.

PSM Yeung, ME Sanders, PM Kitts, R Cano, PS Tong (2002). Species-specific identification of commercial probiotic strains. J Dairy Sci, 85:1039-1051.

Sorathia, N., \& S Rajadhyaksha, M. (2016). Caenorhabditis elegans: A Model for Studying Human Pathogen Biology. Recent Patents on Biotechnology, 10(2), 217-225.

Tomasz Gosiewski \& Agnieszka Chmielarczyk, Magdalena Strus, Monika Brzychczy-Włoch, Piotr B. Heczko (2012). The application of genetics methods to differentiation of three Lactobacillus species of human origin. Ann Microbiol, 62:1437-1445.

Wilson M (2005). Microbial Inhabitant of Humans: Their ecology and role in health and disease. Cambridge University Press.

Yasuda, M., \& Shiaris, M. P. (2005). Differentiation of bacterial strains by thermal gradient gel electrophoresis using non-GC-Clamped PCR primers for the 16S-23S rDNA intergenic spacer region. FEMS Microbiology Letters, 243(1), 235-242.

Zhang, J., \& Liu, X. (2002). Study on tetrazolium salt colorimetric assay for growth and survival of bacteria. Wei sheng yan jiu= Journal of hygiene research, 31(5), 361-363. 